\title{
Efecto de las nuevas tecnologías de la información y comunicación (TICs) en la agilidad mental de las personas de las personas mayores. Estudio Piloto ${ }^{a}$.
}

\author{
Gabriel Medianero ${ }^{1}$, Abdel A. Solís Rodríguez ${ }^{2, *}$ y Biseth \\ Arauz Arauz ${ }^{3}$ \\ ${ }^{1}$ Estudiante, $10^{\circ}$ The Oxford School, Panamá, República de Panamá \\ ${ }^{2}$ Investigador, Facultad de Ciencias Sociales, Universidad Católica Santa María la Antigua (USMA), Panamá, \\ Panamá. \\ ${ }^{31}$ Mentora científica, Facultad de Biociencias y Salud Pública, Universidad Especializada de las Américas, \\ Panamá, Panamá \\ *Autor para correspondencia. Email: asolisr@,usma.ac.pa
}

Recibido: 1 de diciembre de 2015

Aceptado: 9 de diciembre de 2015

\begin{abstract}
The emergence of new information and communications technologies (ICTs) is a reality from which Panama is no exception. People from all age groups must face the challenge of adapting to the use of ICT's. Previous studies have shown that technology can aid in preventing or avoiding depression, by reducing the sense of loneliness. This research aims to identify if the use of ICTs has any impact in cognitive activity in a sample of 51 adults over the age of 50. Participants answered a survey with information about their use of technology. Trail Making Test (parts A and B) was also completed in order to measure psychomotor velocity and mental flexibility. Findings suggests that new technologies have a positive effect on mental agility of people who use new technologies and perceived them as beneficial.
\end{abstract}

Keywords: New technologies; Aging; Psychomotor velocity; Mental flexibility; Trail Making Test.

\footnotetext{
a Trabajo de investigación realizado en el marco del Programa de Jóvenes Científicos organizado por el SENACYT y presentado en la Feria Científica Juvenil, noviembre 2015. Obtuvo el tercer lugar en la categoría "Ciencias Sociales y el Comportamiento".
} 
Invest. pens. crit.

Vol. 3, No. 3, septiembre-diciembre 2015.

pp. 5-

\section{Resumen}

El auge de las Nuevas Tecnologías de las Comunicación (TICs) es una realidad de la que Panamá no escapa. Su uso es un reto al que deben adaptarse las personas de todos los grupos etarios. Estudios previos han demostrado que la tecnología puede ayudar a las personas a manejar o a evitar la depresión, disminuyendo el sentimiento de soledad. Esta investigación buscaba conocer qué beneficios tiene el uso de las TICs en la actividad cognitiva, en un grupo de 51 personas mayores de 50 años. Se aplicó una encuesta de uso de tecnología y la Prueba de Trazo, partes A y B, con el fin de medir la velocidad de procesamiento y la flexibilidad mental. Los resultados aportan evidencia de un efecto positivo en la agilidad mental de aquellas personas que usan las nuevas tecnologías y perciben las TICs como beneficiosas y recomendables.

Palabras clave: Nuevas tecnologías; Envejecimiento; Velocidad de procesamiento; Flexibilidad mental; Prueba de Trazo.

\section{Introducción}

En la actualidad, es evidente el creciente auge en el uso de las nuevas Tecnologías de la Información y la Comunicación (TICs) en todas las etapas de la vida. Nuestro país no se escapa de esta realidad y, según estadísticas de la Unión Internacional de Telecomunicaciones (ITU, 2015), casi el 50\% de la población hace uso del Internet y un $76 \%$ de personas están en contacto con el teléfono celular. Estas cifras reflejan el importante papel que juegan las TICS en la vida diaria de las personas, tanto para la comunicación, formación y vida social (Pavón-Ravasco, 2000). Las TICs van transformando muchas de las facetas en que se desenvuelven las personas, ya sea el hogar, el trabajo, la salud, los ambientes físicos y hasta influye en la calidad de vida (AGE, 2008).

Se observa un incremento en la cantidad de personas mayores de 50 años que entran en contacto con las TICs. Aunque su incursión resulte más lenta, muchos adultos parecen estar cada vez más en modo "online" (Sebastián Morillas \& Martínez Navarro, 2013), ampliando su círculo de amistades independientemente de los idiomas o el lugar (Pavón-Ravasco, 2000). Por tanto, se podrían considerar a las TICs como una herramienta útil para recibir y proporcionar apoyo social, enfrentar la soledad, mejorar la sensación de auto-eficacia en esta etapa de la vida (Leist, 2013). Es indispensable que se profundice en el estudio de los beneficios del uso de TICs en personas mayores, más allá de la mejora en contacto social y acceso a la información (Morris et al., 2014).

Ahora bien, esta realidad reflejada en cifras y en lo que a diario se observa sobre el uso de las TICs, plantea una variable ambiental que podría estar influyendo en la actividad cognitiva. El uso de las TICS requiere de esfuerzo cognitivo, la atención y el aprendizaje; una actividad que ha sido denominada como multitarea (multitask). La multitarea favorece la flexibilidad mental, cambiando la forma de los procesos de aprendizaje y memoria (M. Courage, 2015; Lui \& Wong, 2012; Maclin et al., 2011). En nuestra vida diaria abundan los ejemplos de que las personas pueden, simultáneamente, atender a una conferencia mientras están pendientes de los mensajes de entrada en su celular; hacer tareas mientras se navega internet; hacer deporte mientras se escucha música; etc. 
Ciertamente, el ser humano ha sido multitarea desde hace mucho tiempo, sólo que ahora lo novedoso de la situación es que lo multitarea se ha acelerado en la última década, impregnando lo cotidiano. El auge creciente y veloz de las TICs pareciera indicar que la capacidad cognitiva humana puede poseer procesos simultáneos para integrar, organizar y responder a múltiples estímulos de forma eficiente y productiva (M. L. Courage, Bakhtiar, Fitzpatrick, Kenny, \& Brandeau, 2015)

La capacidad cognitiva necesita de una adecuada capacidad atención para su funcionamiento adecuado. Las actividades multitarea demandan una atención dividida — capacidad de seleccionar de forma flexible, es decir, enfocando y cambiando el foco atencional más relevantes para la actividad y resistir a la distracción de los no relevantes (Posner \& Peterson, 1990). La actividad implica que se realicen simultáneamente dos o más tareas a la vez, donde cada una tiene objetivos distintos, por lo que puede implicar fallos de interferencias (Carrier, Rosen, Cheever, \& Lim, 2015). El tiempo de reacción en la respuesta del sujeto a cada tarea genera una demora por la espera de la respuesta anterior (Pashler, Johnston, \& Ruthruff, 2001). El sustrato neuroanatómico que sustenta el planteamiento ejecutivo en la actividad de doble tarea lo sustentan los estudios de neuroimagen en los que se evidencia que es el área de la corteza prefrontal lateral posterior la que muestra activación en este tipo actividades de multitareas (Dux et al., 2009).

Esta investigación se plantea como una estudio piloto cuyo objetivo principal es conocer si existen diferencias cognitivas en cuando a la atención, la velocidad de respuesta y la flexibilidad cognitiva entre las personas que se consideran usuarios de las TICs en comparación con aquellos que no las utilizan o las usan muy poco. En Panamá, no se encuentran estudios que den luz sobre este tema, por lo que marcaría un precedente sobre la temática y daría pie a realizar estudios más complejos.

\section{Materiales y métodos}

Esta investigación es de diseño observacional y de corte transversal. La muestra estuvo conformada por 51 personas (31 mujeres y 20 hombres) con una media de edad de 61.14 años (desviación típica 7.3 años), todos procedentes de la ciudad de Panamá. La participación fue voluntaria luego de haber sido informados de los objetivos de la investigación y de haber obtenido la firma del consentimiento informado. La muestra se dividió en dos subgrupos: participantes que usan TICs ( $\mathrm{n}=38$ ) y participantes que no usaban TICs ( $\mathrm{n}=13)$. A todos los participantes se les aplicó un cuestionario sobre el uso de las nuevas tecnologías y calidad de vida, elaborado por el equipo de investigación. Luego se les aplicó la Prueba del Trazado, que es un test que se podría considerar como un indicador de la velocidad de procesamiento o velocidad grafomotra y atención, en el desempeño de la Parte A, y a la vez podría ser un indicador del Funcionamiento Ejecutivo (flexibilidad cognitiva, inhibición) en el desempeño de la Parte B (Llinas-Regla et al., 2015). Los datos fueron analizados con la prueba no paramétrica de MannWhitney para dos grupos independientes, debido a que las distribuciones no cumplían el requisito de normalidad. No se cumplió con el requisito de normalidad para aplicar una prueba paramétrica, por

lo que se procede a aplicar su homónima no paramétrica. Además, se realizó un análisis descriptivo y correlacional de los resultados de la encuesta sobre el uso de TICs. La relación entre el uso de las TICS 
Invest. pens. crit.

Vol. 3, No. 3, septiembre-diciembre 2015.

pp. 5-

y las variables sociodemográficas se analizó con el estadístico V de Kramer (en el caso del Nivel educativo y el Sexo) y la prueba t de Student dos grupos independientes (en el caso de la Edad). Todos los procedimientos de la investigación fueron tratados según los estándares éticos y de confidencialidad que rigen actualmente.

\section{Resultados y discusión}

En la Tabla 1 se muestran las medias de las puntuaciones obtenidas por los participante en el estudio en el TMT, parte A y B, agrupados en las dos ramas del estudio, los que sí usan las TICs y los que no. En el caso de la Parte A se puede observar que es mayor el tiempo de los que no usan tecnologías y menor los que la usan. Al aplicar la prueba de contraste no paramétrica, se encuentran diferencias significativas $(\mathrm{p} \leq 0.002)$ en la agilidad mental: la velocidad de procesamiento mental de las personas que utilizan nuevas tecnologías es mayor que la de aquellos no lo las utilizan.

Tabla 1.

Resultados en la prueba del trazado en los dos grupos de estudio.

\begin{tabular}{ccccccccc}
\hline \multirow{2}{*}{ ¿Usa usted TICs? } & \multicolumn{4}{c}{ TMT Parte A } & \multicolumn{4}{c}{ TMT Parte B } \\
\cline { 7 - 10 } & $\boldsymbol{n}$ & $\boldsymbol{M d n}$ & $\boldsymbol{M}$ & $\boldsymbol{D T}$ & $\boldsymbol{M d n}$ & $\boldsymbol{n}$ & $\boldsymbol{M}$ & $\boldsymbol{D} \boldsymbol{T}$ \\
\hline No & 13 & 92.0 & 78.9 & 37.0 & 223.0 & 13 & 226.6 & 85.1 \\
Sí & 38 & 34.0 & 39.3 & 23.3 & 87.8 & 38 & 95.3 & 53.2 \\
\hline Total & $\mathbf{5 1}$ & $\mathbf{3 6 . 8}$ & $\mathbf{4 9 . 4}$ & $\mathbf{3 2 . 2}$ & $\mathbf{1 0 0 . 0}$ & $\mathbf{5 1}$ & $\mathbf{1 2 6 . 0}$ & $\mathbf{8 3 . 0}$ \\
\hline
\end{tabular}

Notas: Número de participantes $(n)$, Mediana $(M d n)$, Media aritmética $(M)$, Desviación

Típica $(D T)$

En el caso de los resultados de la parte B, también se encuentran diferencias significativas entre los grupos de estudio. Las personas que utilizan las nuevas tecnologías, además de poseer una buena velocidad de procesamiento mental, pueden verse beneficiadas de un adecuado funcionamiento ejecutivo, con mayor flexibilidad mental, capacidad de atención alternante, buena capacidad de razonamiento y resolución de problemas.

Los resultados en este estudio piloto serían compatibles con el efecto del uso videojuegos en la velocidad de respuesta (Dye, Green, \& Bavelier, 2009), control atencional (Cardoso-Leite et al., 2015) o que el uso de las TICs beneficia cognitivamente siempre y cuando sean usadas adecuadamente, controladas por el individuo o las plataformas permitan el aprendizaje y el entrenamiento de la actividad multitarea (M. L. Courage et al., 2015).

$\mathrm{Al}$ analizar las variables sociodemográficas que pudieran influir en el uso de las TICs, los resultados indican que entre menos nivel educativo poseen las personas, están menos propensas a usar las tecnologías (Figura 1). Por el contrario, estarán más en contacto con la tecnologías las personas que poseen un mayor nivel educativo, observándose una correlación positiva entre estas dos variables $(\mathrm{V}=.660 \mathrm{p} \leq .000)$. 
Mediante una prueba de hipótesis t de Student, se encuentra evidencia que sí parece existir una posible asociación entre la edad y el uso de tecnologías $\left(\mathrm{t}_{49}=2.613, \mathrm{p} \geq .120, \mathrm{r}=.341\right)$. Estos resultados se pueden ver gráficamente en la Figura 2 donde hay una tendencia a la baja en el uso de TICs a medida que pasan los años.

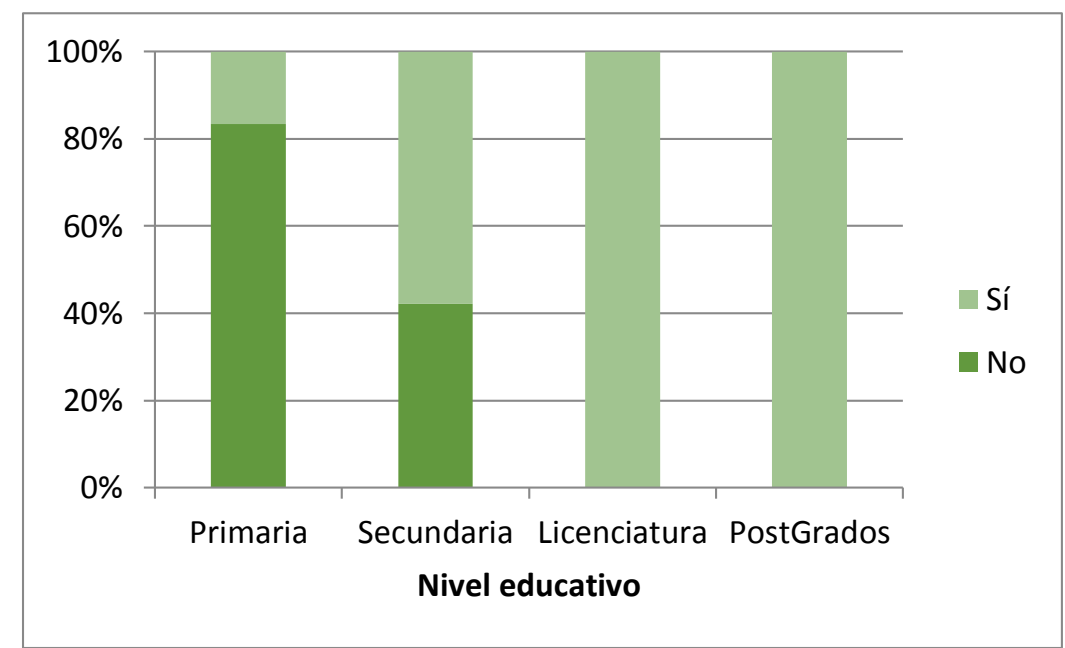

Figura 1. Uso de TICs en función del nivel educativo alcanzado por los participantes.

Tal como se muestra en la Figura 1, el sexo no parece influir en el uso de las TICs (V=.101 p $\geq .470)$.

De manera general, el $92.2 \%$ de los participantes en la investigación consideran que las TICs ayudan a mejorar varias de sus capacidades mentales. El 68.6\% considera que ayudan a mejorar las relaciones con otros mayores, en contraste con un $78.4 \%$ que considera que las TICs ayudan a mejorar las relaciones con los más jóvenes. Con base en su experiencia, el $92.2 \%$ de los encuestados recomendarían a los demás el uso de las TICs en la vida diaria y están en desacuerdo (87.3\%) con abandonar las TICs y seguir como antes.

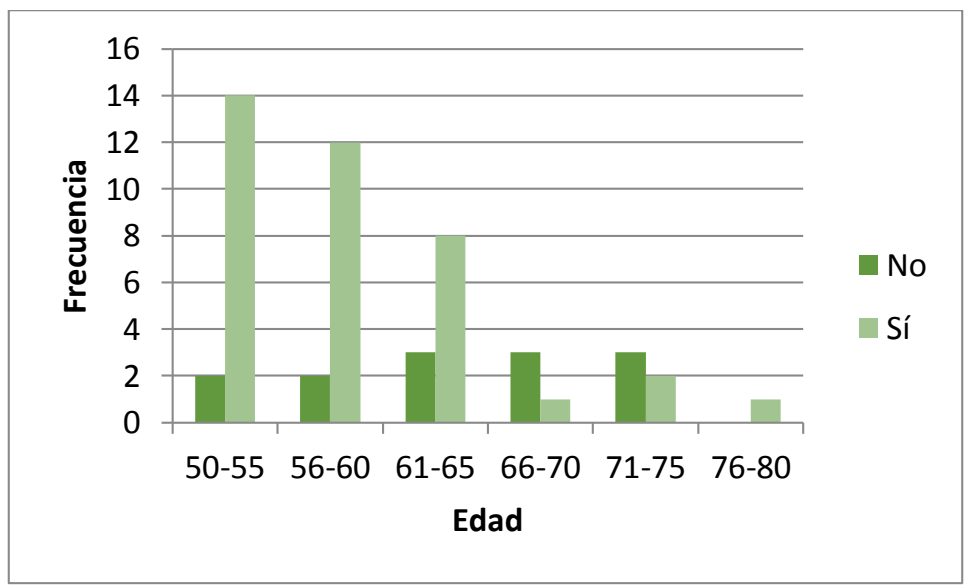

Figura 2. Uso de TICs en función de la edad de los participantes. 
Invest. pens. crit.

Vol. 3, No. 3, septiembre-diciembre 2015.

pp. 5-

Estos resultados parecieran constatar el hecho de las personas mayores cada vez más hacen uso de las TICs y ven en ellas actividades de las cuales se podrían beneficiar (Leist, 2013). Sin embargo, también hay que tener presente que la educación puede ser un factor que genere una brecha tecnológica entre las personas mayores, provocando su abandono (Olphert \& Damodaran, 2013). En este sentido, otra investigación (Elliot, Mooney, Douthit, \& Lynch, 2014) señala también que la edad, el factor socioeconómico y la capacidad cognitiva predisponen el uso de las TICs, y pone de relieve el hecho de que no necesariamente el uso de las TICs aumentan la salud mental o el bienestar, pero sí podrían proteger contra los síntomas depresivos.

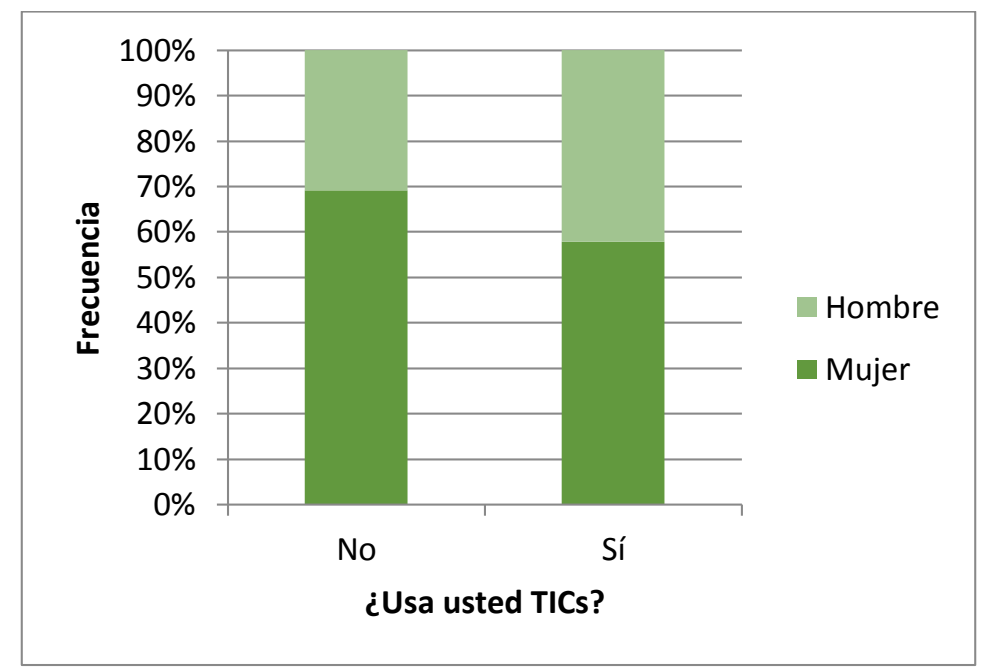

Figura 3. Uso de las TICs en función del sexo de los participantes.

Los resultados de este estudio tienen un alcance exploratorio y no pretenden ser representativos de la población panameña. Sin embargo, abren el campo a una nueva línea de investigación en el país. Se ha encontrado evidencia de que el uso de la tecnología parece favorecer la mejora en las capacidades cognitivas, específicamente en la agilidad mental y las funciones ejecutivas. Además, se observa que existe una percepción que el contacto con las nuevas tecnologías favorece las capacidades mentales, las relaciones sociales e intergeneracionales, y además tienen un buen nivel de aceptación, tanto así que recomendarían su uso.

\section{Agradecimientos}

Un agradecimiento especial a las colaboradoras de la Dirección de Gestión de Ciencia y Tecnología de la SENACYT, quienes fueron las responsables del Programa de Jóvenes Científicos 2015.

\section{Referencias}

AGE. (2008). Media literacy, digital exclusion and older people. The European Older People's Plataform, 2015, Disponible en:

http://www.age-platform.eu/age-publications-and-other-resources/age-publications 
Cardoso-Leite, P., Kludt, R., Vignola, G., Ma, W. J., Green, C. S., \& Bavelier, D. (2015). Technology consumption and cognitive control: Contrasting action video game experience with media multitasking. Atten Percept Psychophys. http://dx.doi.org/10.3758/s13414-015-0988-0

Carrier, L. M., Rosen, L. D., Cheever, N. A., \& Lim, A. F. (2015). Causes, effects, and practicalities of everyday multitasking. Developmental Review, 35, 64-78.

http://dx.doi.org/10.1016/j.dr.2014.12.005

Courage, M. (2015). Translational science and multitasking: Lessons from the lab for the everyday world. Developmental Review, 35. http://dx.doi.org/10.1016/i.dr.2014.12.007

Courage, M. L., Bakhtiar, A., Fitzpatrick, C., Kenny, S., \& Brandeau, K. (2015). Growing up multitasking: The costs and benefits for cognitive development. Developmental Review, 35, 5-41. http://dx.doi.org/10.1016/j.dr.2014.12.002

Dux, P. E., Tombu, M. N., Harrison, S., Rogers, B. P., Tong, F., \& Marois, R. (2009). Training improves multitasking performance by increasing the speed of information processing in human prefrontal cortex. [Research Support, N.I.H., Extramural]. Neuron, 63(1), 127-138. http://dx.doi.org/10.1016/i.neuron.2009.06.005

Dye, M. W., Green, C. S., \& Bavelier, D. (2009). Increasing speed of processing with action video games. Curr Dir Psychol Sci, 18(6), 321-326. http://dx.doi.org/10.1111/j.1467-8721.2009.01660.x

Elliot, A. J., Mooney, C. J., Douthit, K. Z., \& Lynch, M. F. (2014). Predictors of older adults' technology use and its relationship to depressive symptoms and well-being. J Gerontol B Psychol Sci Soc Sci, 69(5), 667-677. http://dx.doi.org/10.1093/geronb/gbt109

ITU. (2015). ITU World Telecommunication /ICT Indicators database. Statistics. International Telecommunication Union. United Nations. Disponible en: http://www.itu.int/en/ITU-D/Statistics/Pages/stat/default.aspx

Leist, A. K. (2013). Social media use of older adults: a mini-review. [Research Support, Non-U.S. Gov't Review]. Gerontology, 59(4), 378-384. http://dx.doi.org/10.1159/000346818

Lui, K., \& Wong, A. (2012). Does media multitasking always hurt? A positive correlation between multitasking and multisensory integration. Psychonomic Bulletin and Review, 19, 647-653.

Llinas-Regla, J., Vilalta-Franch, J., Lopez-Pousa, S., Calvo-Perxas, L., Torrents Rodas, D., \& GarreOlmo, J. (2015). The Trail Making Test: Association with other neuropsychological measures and normative values for adults aged 55 years and older from a spanish-speaking populationbased sample. Assessment. http://dx.doi.org/10.1177/1073191115602552 
Invest. pens. crit.

Vol. 3, No. 3, septiembre-diciembre 2015.

pp. 5-

Maclin, E. L., Mathewson, K. E., Low, K. A., Boot, W. R., Kramer, A. F., Fabiani, M., \& al., e. (2011). Learning to multitask: Effects of video game practice on electrophysiological indices of attention and resource allocation. Psychophysiology, 48, 1173-1183.

Morris, M. E., Adair, B., Ozanne, E., Kurowski, W., Miller, K. J., Pearce, A. J., . . Said, C. M. (2014). Smart technologies to enhance social connectedness in older people who live at home. [Research Support, Non-U.S. Gov't Review]. Australas J Ageing, 33(3), 142-152. http://dx.doi.org/10.1111/ajag.12154

Olphert, W., \& Damodaran, L. (2013). Older people and digital disengagement: a fourth digital divide? [Research Support, Non-U.S. Gov't]. Gerontology, 59(6), 564-570. http://dx.doi.org/10.1159/000353630

Pashler, H., Johnston, J. C., \& Ruthruff, E. (2001). Attention and performance. Annual Review of Psychology, 53, 629-651.

Pavón-Ravasco, F. (2000). Tecnologías avanzadas : nuevos retos de comunicación para los mayores. Revista Iberoamericana de comunicación y educación., 12.

Posner, M. I., \& Peterson, S. E. (1990). The attention systems of the human brain. Annual Review of Neuroscience, 13, 25-42.

Sebastián Morillas, A., \& Martínez Navarro, G. (2013). La influencia de las nuevas tecnologías: videojuegos, redes sociales e internet, en los consumidores seniors en España. Paper presented at the I Congreso Internacional de Comunicación y Sociedad Digital Logroño, España. 